\title{
Computed tomography and magnetic resonance features of intracranial hemangioendothelioma: A study of 7 cases
}

\author{
WEI-ZHONG TIAN $^{1 *}$, XIANG-RONG YU ${ }^{2 *}$, WEI-WEI WANG ${ }^{3}$, BO ZHANG $^{3}$, JIAN-GUO XIA $^{1}$ and HAN-QIU LIU ${ }^{3,4}$ \\ ${ }^{1}$ Department of Radiology, Taizhou People's Hospital, Taizhou, Jiangsu 225300; ${ }^{2}$ Department of Radiology, \\ Zhuhai People's Hospital, Jinan University, Zhuhai, Guangdong 519000; ${ }^{3}$ Department of Radiology, \\ Huashan Hospital, Fudan University, Shanghai 200040; ${ }^{4}$ Department of Radiology, \\ Huashan Hospital Baoshan Branch, Fudan University, Shanghai 200431, P.R. China
}

Received January 7, 2015; Accepted February 4, 2016

DOI: $10.3892 / \mathrm{ol} .2016 .4356$

\begin{abstract}
The current study aimed to present the neuroradiological and histopathological features of intracranial hemangioendothelioma (HE). The computed tomography (CT; $\mathrm{n}=3$ ) and magnetic resonance imaging (MRI; $n=7)$ features, and the clinical presentations of 7 patients with pathologically documented HEs were retrospectively analyzed. Lesions were observed in the right side of the skull (the frontal bone in 1 patient and the parietal bone in 1 patient), the tentorium ( 2 patients), the cerebral falx (1 patient), the right cavernous sinus (1 patient) and the right temporal lobe (1 patient). The tumor was lobulated in 5 cases and round in 2 cases. The majority of tumors appeared isointense or hypointense with multiple scattered hyperintensities on T1-weighted MRI. Moreover, the lesions appeared as inhomogeneous hyperintense regions with multiple enlarged and tortuous blood flow voids on T2-weighted MRI. The lesions also showed marked gadolinium enhancement in a honeycomb pattern. CT scan results showed a isoattenuation region (32-47 HU), with numerous small, round, high-density foci. The 2 cases with skull lesions presented with local bone destruction and discontinuous bone lines of the tabula interna ossis cranii. In 1 case, MR angiography revealed abnormal vessels in the basilar region. A total of 4 cases were epithelial HE, 2 were retiform $\mathrm{HE}$ and 1 was kaposiform HE. Histological examination revealed endothelial cell proliferation with vascular lesions and a mucous matrix
\end{abstract}

Correspondence to: Mr. Han-Qiu Liu, Department of Radiology, Huashan Hospital, Fudan University, 12 Wulumuqi Middle Road, Shanghai 200040, P.R. China

E-mail: drhancher@163.com

Mr. Jian-Guo Xia, Department of Radiology, Taizhou People's Hospital, 210 Yingchun Road, Taizhou, Jiangsu 225300, P.R. China E-mail: drxiajg@126.com

"Contributed equally

Key words: brain tumor, hemangioendothelioma, computed tomography, magnetic resonance imaging or dense fibrous mesenchyme. In conclusion, intracranial HE is rare, but should be considered in the differential diagnosis when evaluating intracranial neoplasms. A well-defined lobulated mass and imaging features that include internal heterogeneity, small scattered hemorrhages and thromboses, signal voids of vessels, and marked and delayed enhancement may confirm the diagnosis of HE.

\section{Introduction}

Intracranial hemangioendothelioma (HE) is a rare borderline angiomatous tumor with invasive behavior and an intermediate level of malignancy between that of benign hemangioma and high-grade malignant angiosarcoma (1).Intracranial epithelioid $\mathrm{HE}$ accounts for $<0.02 \%$ of all primary intracranial tumors, and the tumor usually develops in the dura mater, cranium and brain. HE involving intracranial structures occasionally results in serious local compressive symptoms, including cranial nerve palsy or a potentially fatal increase in intracranial pressure. Despite the low proliferation indices, the clinical course of intracranial HE can be complicated (2). A total resection is essential where possible, otherwise radiotherapy and/or chemotherapy are required (2). Pre-operative embolization of the feeding-artery is recommended. As intracranial HE is seldom encountered in clinical practice, considerable confusion exists with regard to its correct diagnosis and management. To date, numerous studies have focused on the histogenetic, histopathological and clinical characteristics of HE (2-11); by contrast, there have been only scattered case studies that have described the imaging findings $(12,13)$. The purpose of the present study was to analyze the computed tomography (CT) and magnetic resonance imaging (MRI) characteristics of intracranial HE, and to describe the pathological features.

\section{Patients and methods}

Patients. This study was approved by the Ethical Review Committee of Huashan Hospital, Fudan University Medical School (Shanghai, China), and the requirement for patient consent was waived for this retrospective study. A retrospective review was performed on 7 patients with surgically and histologically proven HE at Huashan Hospital (Shanghai, 
China) between January 2008 and December 2013. The patients included 1 male and 6 females, with ages ranging from 13 to 62 years and a median age of 51 years at the initial diagnosis.

CT. CT examinations were performed using a CT machine (Somatom Emotion, Siemens, Erlangen, Germany; or LightSpeed, GE Medical Systems, Milwaukee, WI, USA). A conventional axial scan $(120 \mathrm{kV}, 180 \mathrm{~mA}, 512 \times 512$ matrix and a section thickness of $10 \mathrm{~mm}$ ) was performed in 3 cases.

MRI. MRI was performed in all 7 patients. All MRI images were acquired using a 1.5T MRI unit (Signa Excite HD Twinspeed; GE Medical Systems) or a 3T MR scanner (Signa Excite GEMSOC01, GE Medical Systems) with a single-channel head coil. Sagittal and axial, T1-weighted [400-msec repetition time (TR)/15-msec echo time (TE)] spin-echo (SE) images; axial, T2-weighted (3,000-msec TR/119-msec TE) fast SE images; and fluid attenuated inversion recovery (FLAIR; 8,500-msec TR/138-msec TE) images were obtained for unenhanced MRI; such images were observed using a field of view (FOV) of 25-35 cm, an image matrix of $256 \times 128$ or $256 \times 256$, and a section thickness of $8 \mathrm{~mm}$ with a 2-mm gap. Diffusion-weighted (DW) MRI studies were acquired in the axial plane using a single-shot SE echo planar imaging sequence with $b$ values of 0 and $1,000 \mathrm{sec} / \mathrm{mm}^{2}$ in five orthogonal directions. Contrast-enhanced sagittal and axial, T1-weighted, and SE MRI images were obtained after the administration of gadolinium diethylenetriamine pentaacetic acid $(0.1 \mathrm{mmol} / \mathrm{kg}$ body weight; Magnevist, Berlex Laboratories, Berlin, Germany). Intracranial time-of-flight MR angiography (MRA) was performed in 1 case using the following parameters: TR/TE, 20-25/3-7 msec; flip angle, $20^{\circ}$; FOV, 178x200 mm; matrix, 256x196; slice thickness, $1.2 \mathrm{~mm}$ (0.6-mm overlap with the adjacent section); and section slices, 140 .

Data analysis. The pre-treatment CT and MRI studies from the initial presentation were reviewed, and tumor location, size, shape, attenuation, presence of calcification, signal intensity, enhancement characteristics, peritumoral edema and associated hydrocephalus were recorded. Clinical data, such as symptoms and their duration, treatment and pathological results, were also reviewed.

Immunohistochemical staining. The tumor tissues were fixed in 10\% formalin (Beijing Solarbio Science \& Technology Co., Ltd., Beijing, China) and paraffin (Shanghai Hualing Health Machinery Plant, Shanghai, China) embedded for routine processing. For immunohistochemistry, $5-\mu \mathrm{m}$ thick sections were deparaffinized using xylene (Wuxi Yasheng Chemical Co., Ltd. Wuxi, China), rehydrated using a graded ethanol series (100, $95,90,80$ and $70 \%$ ) and treated with $0.3 \% \mathrm{H}_{2} \mathrm{O}_{2}$ to block endogenous peroxidase activity. The sections were then incubated with $10 \mathrm{mmol} / \mathrm{l}$ citrate buffer (pH 6.0; Beijing Solarbio Science \& Technology Co., Ltd.) at $121^{\circ} \mathrm{C}$ for $20 \mathrm{~min}$ in an autoclave for antigen retrieval. After rinsing with phosphate-buffered saline (PBS; Beijing Solarbio Science \& Technology Co., Ltd.), the sections were incubated with $10 \%$ goat serum (Gibco; Thermo Fisher Scientific, Inc., Waltham, MA, USA) for $1 \mathrm{~h}$ at room

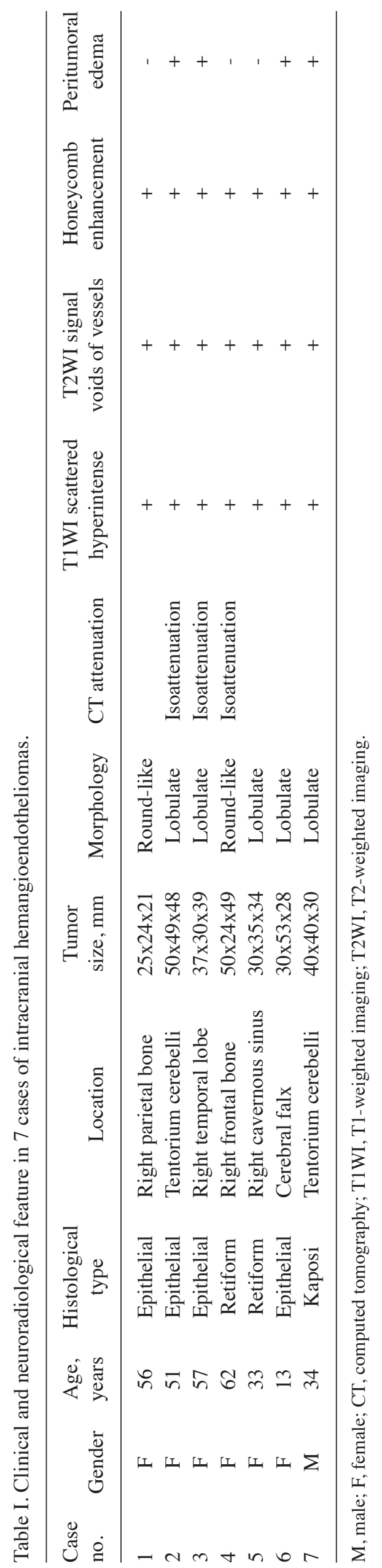


Table II. Immunohistochemistry features and pathological diagnosis of intracranial hemangioendothelioma.

\begin{tabular}{lcccccc}
\hline $\begin{array}{l}\text { Case. } \\
\text { no }\end{array}$ & $\begin{array}{c}\text { Histological } \\
\text { type }\end{array}$ & CD34 & CD31 & FVIII & SMA & Vim \\
\hline 1 & Epithelial & + & - & - & + & - \\
2 & Epithelial & + & + & + & + & + \\
3 & Epithelial & + & - & - & + & - \\
4 & Retiform & + & + & + & - & - \\
5 & Retiform & + & + & + & - & - \\
6 & Epithelial & + & + & - & + & - \\
7 & Kaposi & + & + & - & - & + \\
\hline
\end{tabular}

CD, cluster of differentiation; FVIII, factor VIII; SMA, smooth muscle actin; Vim, vimentin.
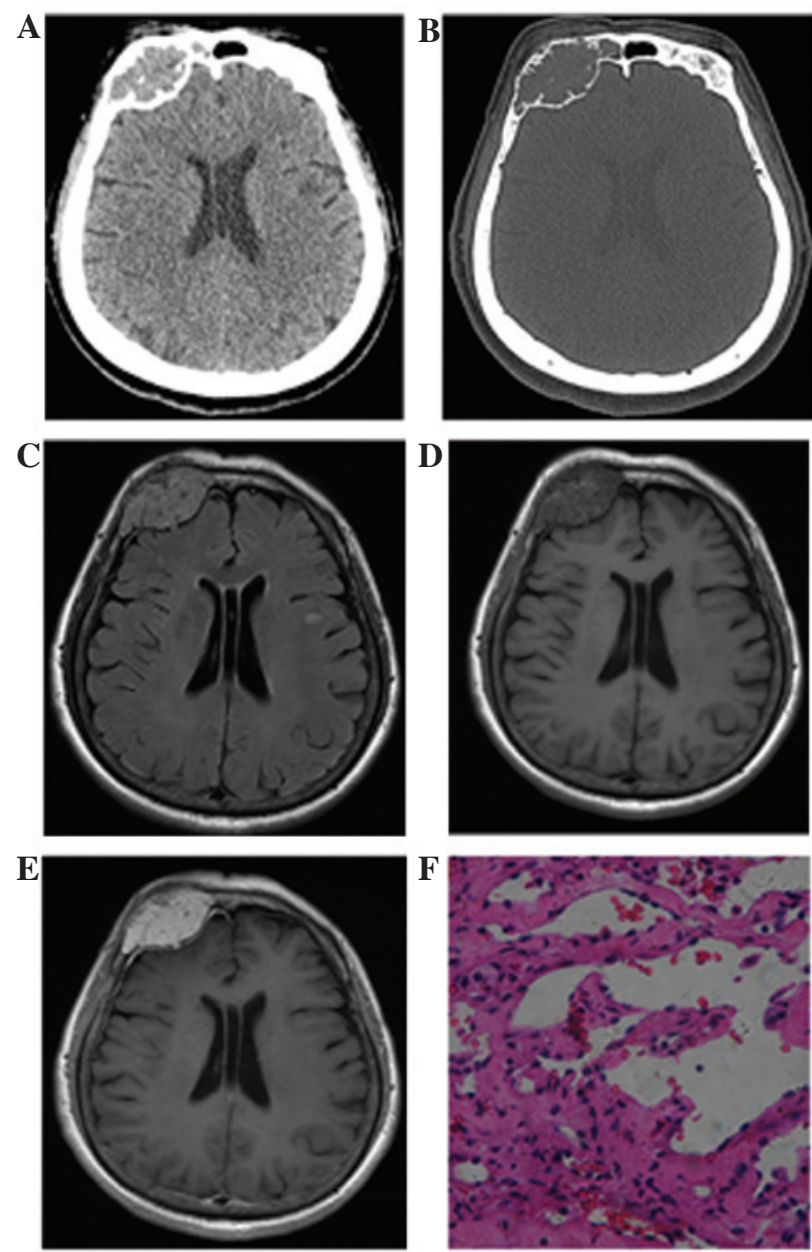

Figure 1. Case 4: A 62-year-old woman with hemangioendothelioma. (A) Axial computed tomography image showing a high-density lesion in the right frontal bone region, with a well-defined border. (B) In the bone window, multiple irregularly thickened trabeculae are noted inside the expansile osteolytic mass, as well as a focal cortical defect. (C) Axial FLAIR images showing a round well-defined mass, with multiple voids of blood vessels, whereas peritumoral edema is absent. (D) Axial T1-weighted images showing a heterogeneous isointense mass with scattered hemorrhage. (E) Axial contrast-enhanced T1-weighted images showing heterogeneous marked enhancement with a dural tail sign. (F) Histopathological examination revealing a tumor composed mainly of plump epithelioid cells, which form primitive vascular channels with areas of prominent vascularity (staining, hematoxylin and eosin; magnification, x100).
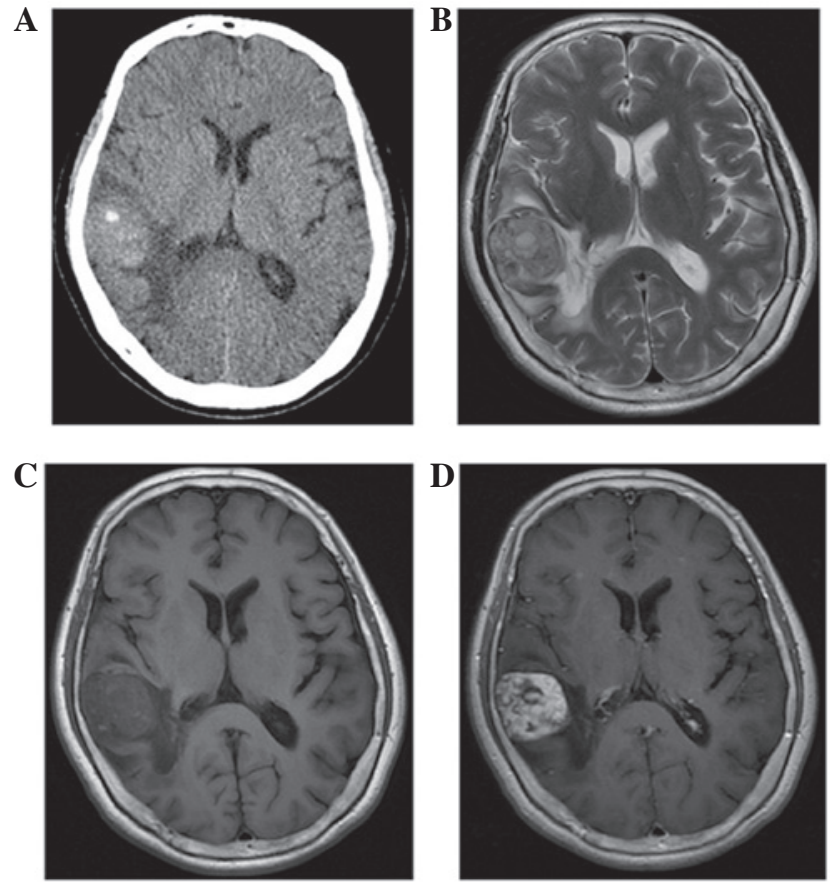

Figure 2. Case 3: A 57-year-old woman with hemangioendothelioma. (A) Axial computed tomography image showing a well-defined high-density mass with a calcified nodule in the right temporal lobe. (B) The lobulated mass is heterogeneous and hyperintense in the axial T2-weighted image, with moderate peritumoral edema. (C) Axial T1-weighted image showing multiple high-intensity dots that reflect hemorrhage and thromboses. (D) Contrast-enhanced axial T1-weighted images showing heterogeneous multi-nodular enhancement.

temperature to block any nonspecific reactions. The sections were then incubated overnight at $4^{\circ} \mathrm{C}$ with mouse anti-human monoclonal glial fibrillary acidic protein (dilution, 1:10; cat. no. M0761), mouse anti-human monoclonal cluster of differentiation (CD)31 (dilution, 1:100; cat. no. IR610), mouse anti-human monoclonal CD34 (dilution, 1:4; cat. no. IR632), rabbit anti-human polyclonal factor VIII (dilution, 1:100; cat. no. A0082), mouse anti-human monoclonal smooth muscle actin (dilution, 1:400; cat. no. IR611) and mouse anti-human monoclonal vimentin (dilution, 1:100; cat. no. IR630) antibodies. The diluted primary antibodies were obtained from Dako (Glostrup, Denmark). Negative control slides were also processed in parallel using a nonspecific immunoglobulin IgG (cat. no. HAB5500149; Sigma-Aldrich, St. Louis, MO, USA) at the same concentration as the primary antibody. Detection of all antibody reactions was performed using a streptavidin peroxidase detection kit (Dako) according to the manufacturer's protocol. After rinsing in PBS, the peroxidase reaction was visualized by incubating the sections with diaminobenzidine tetrahydrochloride in $0.05 \mathrm{~mol} / \mathrm{l}$ Tris buffer $(\mathrm{pH}$ 7.6) containing $0.03 \% \mathrm{H}_{2} \mathrm{O}_{2}$. After washing in water three times, the sections were counterstained with hematoxylin (Beijing Solarbio Science \& Technology Co., Ltd.), dehydrated, cleared and coverslipped. The slides wer then visualized under an optical microscope (CX31; Olympus Corporation, Tokyo, Japan).

\section{Results}

The main clinical manifestations included headache and dizziness (3 cases), paroxysmal dysesthesia and paresis of the 

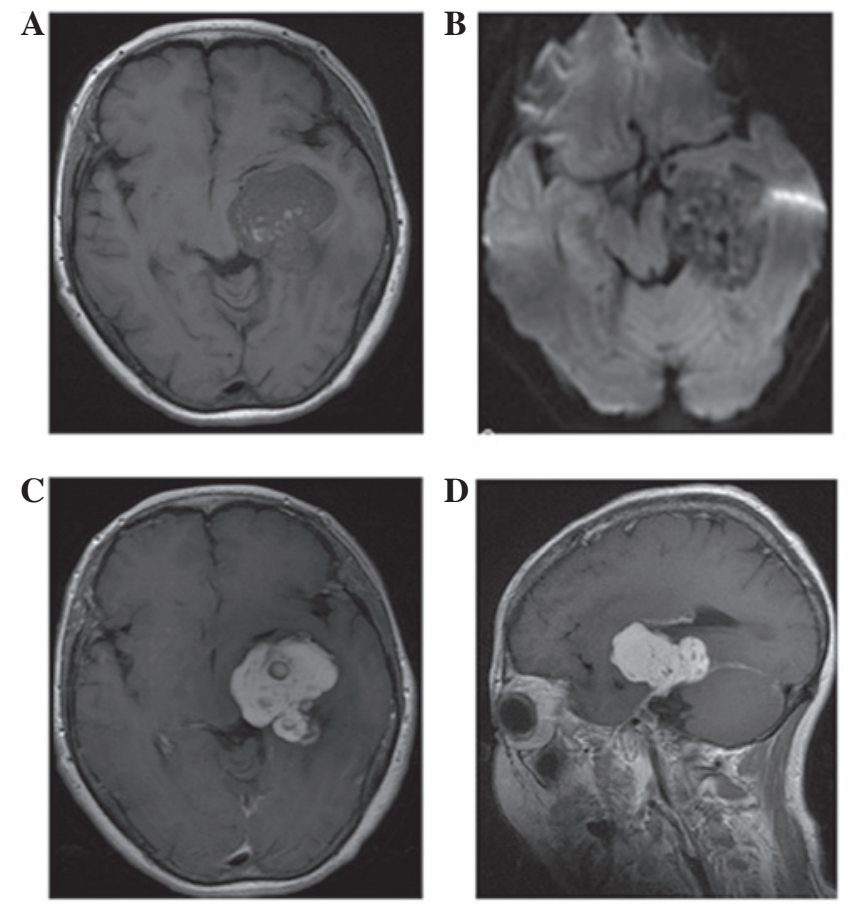

Figure 3. Case 2: Neuroimaging for hemangioendothelioma at the tentorium cerebelli region in a 51-year-old woman. (A) T1-weighted image showing a primarily isointense lobulated mass with scattered hemorrhage. (B) Axial diffusion-weighted image (b-factor: $1,000 \mathrm{~mm} / \mathrm{sec}^{2}$ ) demonstrating a inhomogeneous high-low mixed signal compared with that of the white matter. (C) Axial and (D) coronal contrast-enhanced T1-weighted images showing heterogeneous marked honeycomb enhancement with a dural tail sign.
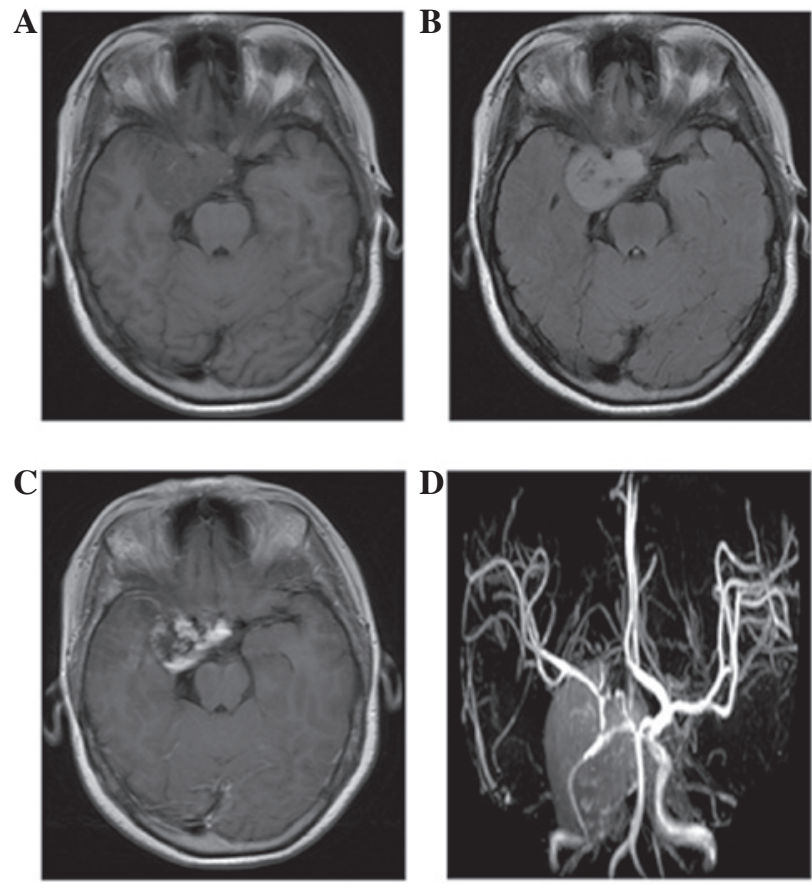

Figure 4. Case 5: A 33-year-old woman with hemangioendothelioma. (A) Magnetic resonance imaging (MRI) findings: The tumor is composed of a marked enhanced solid portion that exhibits hemorrhage and thromboses. The lobulated mass is heterogeneously isointense in the right cavernous sinus on unenhanced axial T1-weighted imaging. (B) Axial fluid attenuated inversion recovery image showing the inhomogeneous hyperintense mass without peritumoral edema. (C) Contrast-enhanced axial T1-weighted image showing heterogeneous marked enhancement. (D) MR angiography sequence showing abnormal multiple vessels in the lesion, which were confirmed by histological examination, that reflect the hypervascular characteristic of the tumor. right limbs (1 case), tinnitus and blurred vision (1 case), and right orbital eminence (1 case). Furthermore, 1 patient was asymptomatic and the lesion was incidentally discovered. Symptom duration ranged between 4 months and 10 years, with a mean of 2 years.

The neuroradiological findings of the HEs are summarized in Table I. While 2 cases exhibited lesions in the right side of the skull, 1 in the frontal bone (Fig. 1) and the other in the parietal bone, another 2 cases presented with lesions in the tentorium. Moreover, 1 case presented with a lesion in the cerebral falx, 1 case with a lesion in the right cavernous sinus and 1 case with a lesion in the right temporal lobe (Fig. 2). CT and MRI results showed that 2 masses were round (Fig. 1), whereas the remaining 5 tumors were lobulated. The total diameter ranged between 2.1 and $5.3 \mathrm{~cm}$. The 3 patients who underwent CT presented predominantly with isoattenuation, with radiodensity values ranging from 32-47 $\mathrm{HU}$, and a dotted calcification was detected in 1 lesion (Fig. 2A). The 2 lesions originating from the skull showed local bone destruction and osteolysis. The cortical bones were discontinuous with sclerotic margins, and exhibited soft-tissue density and spine-like protuberances (Fig. 1A). Moreover, 1 case occurred in the right frontal bone and involved the superior edge of the right orbit and frontal sinus.

The MRI images of the 7 lesions were primarily isointense $(n=5)$ or mildly hypointense $(n=2)$ compared with the gray matter on T1-weighted images. All tumors were scattered hyperintensely due to intratumoral hemorrhage (Figs. 1D, 2C, 3A and 4A). T2-weighted and FLAIR images showed that all tumors were inhomogeneously hyperintense to the cortex. Mild or moderate peritumoral edema was noted in 4 cases, whereas no evident edema signals were observed in the 2 cases with skull lesions and in the case with a lesion in the right cavernous sinus (Fig. 4). The lesions exhibited a lobulated and septated appearance due to the coalescence of multiple high-signal nodules within the tumor (Fig. 2B). The signal voids in the vessels were also visible in all cases (Figs. 1C, 2B and 4B). DW imaging $(b=1,000)$ showed that 6 tumors were hypointense, whereas 1 tumor was inhomogeneous with high-low mixed signal compared with that of the white matter (Fig. 3B).

Contrast-enhanced T1-weighted images showed contrast enhancement in all tumors. All lesions exhibited inhomogeneous enhancement consisting of multiple nodules with relatively homogeneous enhancement compared with the surroundings in the early stage; such nodules became hyperintense with delayed enhancement. Additionally, 4 cases demonstrated a marked enhancement, called the 'dural tail sign' in the dura adjacent to the tumor. MRA also revealed ectopic vessels in the basilar region in 1 case (Fig. 4C).

A total resection was performed in 5 patients, whereas a partial resection was performed in the remaining 2 patients, including the case with the skull invading the dura. Light microscopy revealed dense spindle and epithelioid cells with abnormal angiogenesis in 4 cases (cases 1-3 and 6). Tubular structures and spindle cell proliferation were also observed in certain regions. An abnormal distribution of endothelial cells within the lesion and a branching 'netlike' vascularization extending into the reticular formation was observed in 2 cases (cases 4 and 5). A single case (case 3) presented with endothelial 
cells showing evident atypia and vacuoles. Moreover, another case (case 7) exhibited loosely-arranged spindle-shaped tumor cells with abundant cleft-like or cribriform small blood vessels in between, but no evident nuclear atypia or mitoses. Thrombogenesis was also observed in certain vascular proliferations (Fig. 1F). The immunohistochemistry results and pathological diagnosis of the HEs are summarized in Table II.

\section{Discussion}

HE occurs in the soft tissues of the limbs, liver, lungs, chest wall or skin. The tumor is rare, and various clinical, radiological and pathological features have been sporadically reported (2-16). To the best of our knowledge, the 7 intracranial HE cases enrolled in the current study represent the largest documented case series. The different forms of HE include retiform, kaposiform, epithelioid, epithelioid sarcoma-like and composite $\mathrm{HE}$, as well as endolymphatic papillary angioendothelioma (17). The incidence of intracranial epithelioid HE accounts for $<0.02 \%$ of all primary intracranial tumors $(5,6)$, whereas retiform and kaposi $\mathrm{HE}$ are even rarer $(7,8)$. In the present study, epithelial-type HE was more common (4 cases) than HE of the retiform (2 cases) or kaposi (1 case) types. The endothelial nature of HE is indicated by the histological features of the tumor and positive staining for endothelial markers, such as CD34, CD31 and factor VIII-related antigen.

HE can affect adults and children, with no clear gender preponderance $(13,14,18,19)$, However, the gender distribution in the present study was $6: 1$, females to males. Clinical symptoms were associated with the location of the lesion, whereas common symptoms included headaches and intracranial hypertension. The tumors often grew slowly; thus, the period from the onset of the first symptom to the time of diagnosis was relatively long. In the present study, the duration of symptoms ranged between 4 months and 10 years. HE is a low-proliferation borderline angiomatous tumor with malignant behavior, such as local recurrence and metastasis (17). Five cases exhibited a lobulated appearance, with different margin intensities from the lesion and the surrounding normal brain tissues. Approximately $50 \%$ of the intra-axial lesions presented with surrounding edema, with mild to moderate edema around the lesion observed in 4 cases. The 2 cases that originated from the skull showed expansive bone destruction. However, the cortical bones of the inner and outer table of the skull were discontinuous, which suggested that these 2 tumors had potential malignant biological behavior.

Histopathological examination revealed the different components of the myxoid matrix within the tumor, and the varying distribution patterns of the tumor cells and mucus matrix components. The majority of the abnormal blood vessels within the tumor were thin-walled ectatic vessels, with lumens of varying diameter containing red blood cells and thromboses. In the present study, all tumors appeared as mildly hypointense to isointense on T1-weighted images and inhomogeneously hyperintense on T2-weighted images compared with that of the gray matter. Areas with low-signal intensity or no signal on T1- and T2-weighted images represented tumor vessels. Furthermore, MRA examination showed abnormal vascularity in the tumor. Zhang et al (12) previously reported abnormal vessels in the basilar region of the lesion using CT angiography.
All cases in the present study exhibited a multiple fleck pattern or smaller punctate hyperintensities on the T1-weighted images, which may be features of HEs that could promote correct imaging assessment. Histological examination revealed scattered small hemorrhages and thromboses in these tumors. Zheng et al (2) reported hemorrhage in approximately one-quarter of intracranial HE lesions. Moreover, Ibarra et al (15) proposed that the high signal in the T1-weighted images is due to the methemoglobin component of the intravascular thrombi. Another characteristic MRI finding in HEs is a high incidence of multiple nodular high-signal lesions with septations on T2-weighted images. The present results are consistent with the previous study by Bourekas et al (20), which demonstrated the slow reflow phenomenon or blood sinus structure within these lesions $(9,16)$.

All 7 HEs in the present study showed tumor enhancement, with the lesions demonstrating heterogeneous enhancement patterns. Histology revealed that the margins were the most active areas of cell proliferation and contained abundant vasoganglion forming blood sinus-like structures, whereas the central region was a solid zone with few blood vessels; this finding may explain the heterogeneous enhancement of the tumors $(6,11,13,21-23)$. The delayed enhancement is possibly associated with the abnormal proliferation of blood vessels and sinus-like structures. The appearance of rich vascularity provides important information for the differential diagnosis and treatment strategy. A dural tail sign was visible in 4 cases based on the invasive growth pattern of the tumor.

DW imaging, initially used in stroke imaging, has been used to study neoplasia. The current study presented HE lesions with low signals during DW imaging, which suggests the presence of loose connective tissue spaces and lower cell components compared with those of the other solid tumors (24). However, the use of DW MRI in differentiating between the histopathological subtypes of HEs has not been well described, and its value in forming a differential diagnosis should be evaluated in large studies.

CT of HE in the skull usually shows a slightly higher density due to the abundant blood sinuses and small amount of bleeding between tumor tissues (16). The two cases that originated from the skull showed extensive bone destruction and osteolysis, as well as discontinuous cortical bones with sclerotic margins; these findings suggest a potentially invasive growth characteristic. Moreover, the observed sparse trabecula bone shadows or spine-like protuberances with evident edges and apparent enhancements resembled those reported in previous studies (14-16). Tumor calcification and periosteal reactions are rarely observed (25). The two cases described in the current study presented with expansion of bone due to involvement of the diploic space, and enhancement, which is similar to the results of previous studies (14-16).

Differential diagnosis for HE usually includes meningioma, hemangioma and angiosarcoma $(12,23)$. The signal intensity on the T2-weighted images was low, which may be one of the main features of meningiomas, whereas the dural tail sign can enable correct imaging assessment. Adjacent skull thickening in meningiomas is a feature of these extra-axial tumors, which is less common in HEs. Hemangioma exhibit a hive-like or fence-like structure, with a rapid enhancement at the early stage and homogeneous enhancement after a delay (15). Primary 
angiosarcoma of the central nervous system is an extremely rare malignancy, which exhibits rapid growth (26). Imaging studies characteristically show a well-demarcated lesion of the cerebral hemisphere with avid enhancement $(26,27)$, thus differentiation from HE is difficult. However, by contrast to HE, angiosarcomas usually present as a heterogeneous mass with significant vasogenic edema and intratumoral cyst formation $(26,27)$.

In conclusion, $\mathrm{HE}$ is rare, but should be considered as a possible diagnosis when a tumor presents as a lobulated mass, with hemorrhage, signal voids of vessels, a heterogeneous appearance and delayed enhancement; these factors could potentially distinguish HE from other primary brain neoplasms. CT and MRI may be useful in providing an early and accurate diagnosis; each method is important due to the propensity of the tumor for abundant vascularization and low-grade malignant biological behavior.

\section{References}

1. Weiss SW and Enzinger FM: Epithelioid hemangioendothelioma: A vascular tumor often mistaken for a carcinoma. Cancer 50 970-981, 1982

2. Zheng J, Liu L, Wang J, Wang S, Cao Y and Zhao J: Primary intracranial epithelioid hemangioendothelioma: A low-proliferation tumor exhibiting clinically malignant behavior. J Neurooncol 110: 119-127, 2012.

3. Baehring JM, Dickey PS and Bannykh SI: Epithelioid hemangioendothelioma of the suprasellar area: A case report and review of the literature. Arch Pathol Lab Med 128: 1289-1293, 2004.

4. Hurley TR, Whisler WW, Clasen RA, Smith MC, Bleck TP, Doolas A and Dampier MF: Recurrent intracranial epithelioid hemangioendothelioma associated with multicentric disease of liver and heart: Case report. Neurosurgery 35: 148-151, 1994.

5. Hamlat A, Casallo-Quilliano C, Saikali S, Lesimple T and Brassier G: Epithelioid hemangioendothelioma of the infundibular-hypothalamic region: Case report and literature review. J Neurooncol 67: 361-366, 2004.

6. Taratuto AL, Zurbriggen G, Sevlever G and Saccoliti M: Epithelioid hemangioendothelioma of the central nervous system. Immunohistochemical and ultrastructural observations of a pediatric case. Pediatr Neurosci 14: 11-14, 1988.

7. Kubota T, Sato K, Takeuchi H and Handa Y: Successful removal after radiotherapy and vascular embolization in a huge tentorial epithelioid hemangioendothelioma: A case report. J Neurooncol 68: 177-183, 2004.

8. Aditya GS, Santosh V, Yasha TC and Shankar SK: Epithelioid and retiform hemangioendothelioma of the skull bone-report of four cases. Indian J Pathol Microbiol 46: 645-649, 2003.

9. Cho WS, Kim SK, Park SH and Cho BK: Intracranial kaposiform hemangioendothelioma: Proposal of a new malignant variant. J Neurosurg Pediatr 3: 147-150, 2009.

10. Mohan SM, Symss NP, Pande A, Chakravarthy VM and Ramamurthi R: Intracranial epithelioid hemangioendothelioma. Childs Nerv Syst 24: 863-868, 2008.
11. Palmieri G, Montella L, Martignetti A and Bianco AR: Interferon alpha-2b at low doses as long-term antiangiogenic treatment of a metastatic intracranial hemangioendothelioma: A case report. Oncol Rep 7: 145-149, 2000.

12. Zhang J, Wang Y and Geng D: Intracranial epithelioid hemangioendothelioma: An unusual CTA finding in one case. Br J Neurosurg 24: 294-295, 2010.

13. Chan YL, Ng HK, Poon WS and Cheung HS: Epithelioid hemangioendothelioma of the brain: A case report. Neuroradiology 43: 848-850, 2001

14. Parajón A and Vaquero J: Meningel intracranial epithelioid hemangioendothelioma: Case report and literature review. J Neurooncol 88: 169-173, 2008.

15. Ibarra RA, Kesava P, Hallet KK and Bogaev C: Hemangioendothelioma of the temporal bone with radiologic findings resembling hemangioma. AJNR Am J Neuroradiol 22: 755-758, 2001.

16. Kim HL, Im SA, Lim GY, Chun HJ, Lee H, Park HJ and Byun JY: High grade hemangioendothelioma of the temporal bone in a child: A case report. Korean J Radiol 5: 214-217, 2004.

17. Requena L and Kutzner H: Hemangioendothelioma. Semin Diagn Pathol 30: 29-44, 2013.

18. Mosoia L, Mabrut JY, Adham M, Boillot O, Ducerf C, Partensky $\mathrm{C}$ and Baulieux J: Hepatic epithelioid hemangioendothelioma: Long-term results of surgical management. J Surg Oncol 98: 432-437, 2008.

19. Lyburn ID, Torreggiani WC, Harris AC, Zwirewich CV, Buckley AR, Davis JE, Chung SW, Scudamore CH and Ho SG: Hepatic epithelioid hemangioendothelioma: Sonographic, CT and MR imaging appearances. AJR Am J Roentgenol 180: 1359-1364, 2003.

20. Bourekas EC, Cohen ML, Kamen CS, Tarr RW, Lanzieri CF and Lewin JS: Malignant hemangioendothelioma (angiosarcoma) of the skull: Plain film, CT and MR appearance. AJNR Am J Neuroradiol 17: 1946-1948, 1996.

21. Fernandes AL, Ratilal B, Mafra M and Magalhaes C: Aggressive intracranial and extra-cranial epithelioid hemangioendothelioma: A case report and review of the literature. Neuropathology 26: 201-205, 2006.

22. Tancredi A, Puca A and Carbone A: Multifocal cerebral hemangio-endothelioma. Case report and review of the literature. Acta Neurochir (Wien) 142: 1157-1161, 2000.

23. Hodaie M, Becker L, Teshima I and Rutka JT: Total resection of an intracerebral hemangioendothelioma in an infant. Case report and review of the literature. Pediatr Neurosurg 34: 104-112, 2001.

24. Rocha Oliveira PC, Alcantara FP, Souza-Vianna PE and Brito AP: Cerebral epithelioid hemangioendothelioma with thoracic simultaneous involvement: Advanced MRI features. Arq Neuropsiquiatr 70: 637-638, 2012.

25. Wold LE, Unni KK, Beabout JW, Ivins JC, Bruckman JE and Dahlin DC: Hemangioendothelial sarcoma of bone. Am J Surg Pathol 6: 59-70, 1982

26. Hackney JR, Palmer CA, Riley KO, Cure JK, Fathallah-Shaykh HM and Nabors LB: Primary central nervous system angiosarcoma: Two case reports. J Med Case Rep 6: 251, 2012.

27. Cookston M, Cotter GW, Schlitt M and Bastian FO: Primary angiosarcoma of the brain. South Med J 84: 517-20, 1991. 